\title{
A data augmentation methodology for training machine/deep learning gait recognition algorithms
}

Christoforos C. Charalambous

http://www.bicv.org/team/christoforos-charalambous/

Anil A. Bharath

http://www.bicv.org/team/anil-anthony-bharath/

\author{
BICV Research Group \\ Department of Bioengineering \\ Imperial College London \\ London, UK
}

In this paper we propose a data augmentation methodology for training machine/deep learning gait recognition algorithms. While previously published methods generated synthetic data to augment training and/or testing sets [1] or to learn features invariant to certain conditions [2], they have incorporated a very limited number of covariate factors. To our knowledge, this is the first attempt to provide the ability to simultaneously generate synthetic data with so many controllable conditions for gait recognition.

The combination of real motion capture, data preparation, avatar construction and scripted rendering allows synthetic frames to be generated, with almost arbitrary degrees of variation. Figure 1 shows a small sample of the controllable confounding factors that can be generated with the proposed methodology.

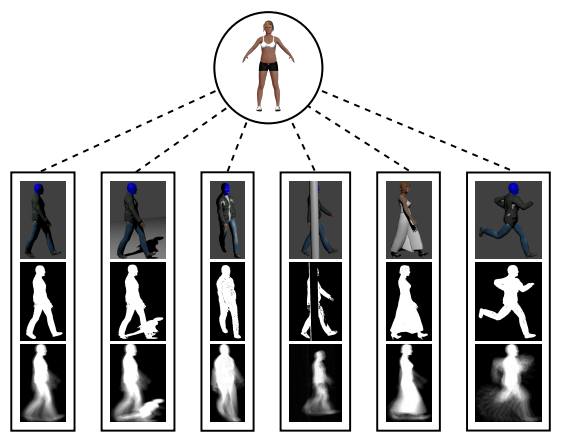

Figure 1: A small sample of the controllable confounding factors. It is readily apparent how the extracted gait features are directly affected.

Using the Gait Energy Image (GEI) [3] as gait features, extracted directly from the synthetically generated frames, we performed experiments to assess the level to which identity is preserved within the synthetic data sets. Results from our experiments - presented in Figure 2 - suggest that information about the identity of subjects is retained within the synthetically generated data. The experiments using GEIs as features within our dataset showed that augment- ing a limited amount of real data with the synthetically generated data can yield identification of subjects with an accuracy of more than $95 \%$. The results of this study suggest that synthetic data can be used to augment the training of gait recognition algorithms provided that the feature set (e.g. principal components) is expanded to include both real and synthetic examples of data.

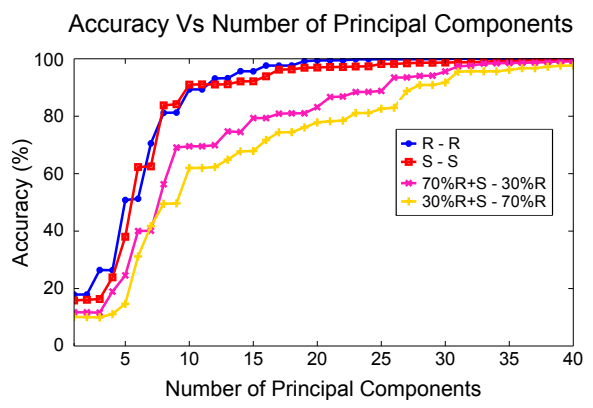

Figure 2: Results from our experiments, with different training and testing sets, showing accuracy achieved with GEI features being projected onto different number of principal components.

The proposed methodology offers the possibility to generate sequences with multiple confounding factors, allowing exploratory work into training machine/deep learning algorithms for fully-invariant gait recognition, with a far greater amount of synthetic training and test data that would otherwise be impossible. The dataset and simulation files will be made publicly available.

[1] J. Shotton et al. Real-time human pose recognition in parts from single depth images. Computer Vision and Pattern Recognition, pages 1297-1304, 2011.

[2] J. Zhang et al. Arbitrary view action recognition via transfer dictionary learning on synthetic training data. IEEE International Conference on Robotics and Automation, page 1678-1684, 2016.

[3] J. Han, B. Bhanu. Statistical feature fusion for gait-based human recognition. Proceedings of the IEEE Computer Society Conference on Computer Vision and Pattern Recognition, 2:0-5, 2004. 